\title{
TELEMAC, a hydroinformatic system
}

\section{Télémac, un système hydroinformatique}

\author{
par J.-M. Hervouet \\ Groupe Hydraulique Fluviale \\ Laboratoire National d'Hydraulique. Direction des Etudes et Recherches. \\ Electricité de France.
}

Cet article est une description générale du système hydroinformatique TELEMAC. On décrit tout d'abord les pré et postprocesseurs qui sont d'une part un mailleur et d'autre part un logiciel de présentation et d'analyse de résultats. Les modules de simulation numérique résolvent tout un ensemble d'équations dans le domaine de la mécanique des fluides à surface libre : équations de Saint-Venant, équations de Navier-Stokes à surface libre, divers modèles de houle, de qualité d'eau et de transport sédimentaire. On aborde ensuite brièvement le sujet du parallélisme avec décomposition de domaines et le couplage externe de codes.

\section{INTRODUCTION}

Through the steady improvements in hardware and algorithms, an ever increasing use of digital modelling can be made in fluid mechanics. Meanwhile, the rising environment-related problems and the concurrent water uses raise more and more complex and multidisciplinary issues. Consequently, the fields of application for numerical simulation are becoming wider, the requirements are ever more exacting. This high rate of development involves significant efforts aiming at four major goals, namely modelling of physical processes, algorithmic investigations, users' interfaces and documentation, and validation.

Such a necessary evolution towards what is sometimes called " hydroinformatics" is costly and the times when computer programmes were only prepared for the duration of a study or a thesis are over. Nowadays, we must work on a long range basis, know how to preserve the acquired experience while rapidly taking advantage of the latest advances in science, making mass production economies, addressing users from various origins, setting up inter-software communications: the idea of a modelling system is being rapidly emerging. At the $\mathrm{LNH}$, and for the free surface flows, the construction of the TELEMAC system is an offspring of these requisite changes.

\section{II - THE TELEMAC SYSTEM}

The TELEMAC software system is a set of computer programs dedicated to the numerical simulation of free surface flows. It is based upon finite element techniques and has been built by Electricité de France. It is also available outside EDF and is currently being used by more than 60 scientific teams in the world, and a majority of hydraulic laboratories in Europe. Other users are the french Navy, water authorities, universities, or consultants. Some universities and engineer schools, such as ENSEEIHT and IMFT in Toulouse, Bristol in England, Hannover in Germany, and Coimbra in Portugal, have also signed co-development agreements with EDF and use the software for education. The system thus benefits from a large community of researchers.

The system is composed on one hand of pre and post-processors, for digitising the data, meshing the computational domain, and displaying the results, and on the other hand of modules solving different free surface flow equations.

The main advantage shared by the various simulation programmes in the TELEMAC system is their high flexibility as imparted by the finite element method in the definition of the flow domain and the local grid refinements. A further advantage is given by the tool kit to be used in the preparation of computations and the graphic interpretation of results.

Furthermore, the various modules also have the same architecture and use common data structures, and are consequently highly compatible. Thus, for example, one can easily switch from a two-dimensional computation to a three-dimensional computation of the flow over a given grid.

We shall give hereafter a brief review of the major pre and post-processors in the system, and then of the numerical simulation programmes. 


\section{III $\square$ PRE AND POST PROCESSORS}

We shall describe briefly the mesh generator MATISSE and the post-processor RUBENS.

MATISSE is a two-dimensional mesh generator based on the knowledge of a digital elevation model. It can thus take into account not only the contour of a domain, but also the bathymetry. As a matter of fact, criterion for the local mesh size can be built interactively and the Delaunay's triangulation algorithm will take them into account. For example it is suitable for maritime studies to have a mesh size proportionnal to the square root of the depth. As far as tidal waves are concerned, it will ensure a constant Courant number in all the domain ; more over an automatic refinement along the coasts is obtained (see figure 1). Given lines called constraint lines can be followed by the mesh, and prescribed given points called hard points can be taken into account and will appear in the final mesh. This mesh generator has been built with the help of the "Ecole des Mines de SaintEtienne "who brought his knowledge of Digital Elevation Models and specific tools for dealing with truncation errors in algorithmic geometry.

The RUBENS software is designed for displaying the results of 1- or 2-dimensional calculations or measurements. It provides a wide range of graphs and allows a quite free paging, in the style of such office tools as MacDraw. In addition to the conventional displays of grids, vector fields, iso-lines, Rubens provides the following:

- spatial profiles in any direction, temporal profiles;

- superimposition of calculations and measurements;

- mouse-controlled zooming, mouse-guided probe giving local data.

Rubens also includes a command interpreter which is designed to form new variables from the results of a calculation. A batch mode is provided for the automation of repetitive jobs or the mass production of images or movies.

\section{IV - SIMULATION PROGRAMMES}

We shall describe the most outstanding features of those programs in 6 chapters:

- TELEMAC-2D: De Saint-Venant (Shallow Water) equations or Boussinesq equations. It has been designed to cope with rapid flows, shocks, floods, dam-breaks, tidal flats and storm surges.

- TELEMAC-3D: Navier-Stokes equations in 3D, with a free surface and a hydrostatic pressure assumption. This model is used in problems involving pollution, thermal dilution, sediment transport and water quality.

- Wave models: ARTEMIS, TOMAWAC and COWADIS. - SISYPHE: bed load transport in 2 dimensions. SISYPHE was developped within the framework of a national program in sediment transport, supported by the ministry of transportation.

- SUBIEF-2D and TELEMAC-3D and their suspended sediment transport applications.

- SUBIEF-2D and 3D to simulate complex water quality models.

All the models are developed in the frame of an EDF's Quality Assurance procedure aiming at providing the product reliability. A complete and detailed documentation is associated with the programme and particularly includes validation specifications.

\subsection{TELEMAC-2D}

TELEMAC-2D computes the non-steady free surface flows in shallow water environments. It makes it possible to study any of the coastal, fluvial, estuarial and lacustrine domains. It can take the following processes into account:

- flow induced by a tide, a flood, a river water intake, a dam failure;

- effects of meteorological events: atmospheric pressure and wind;

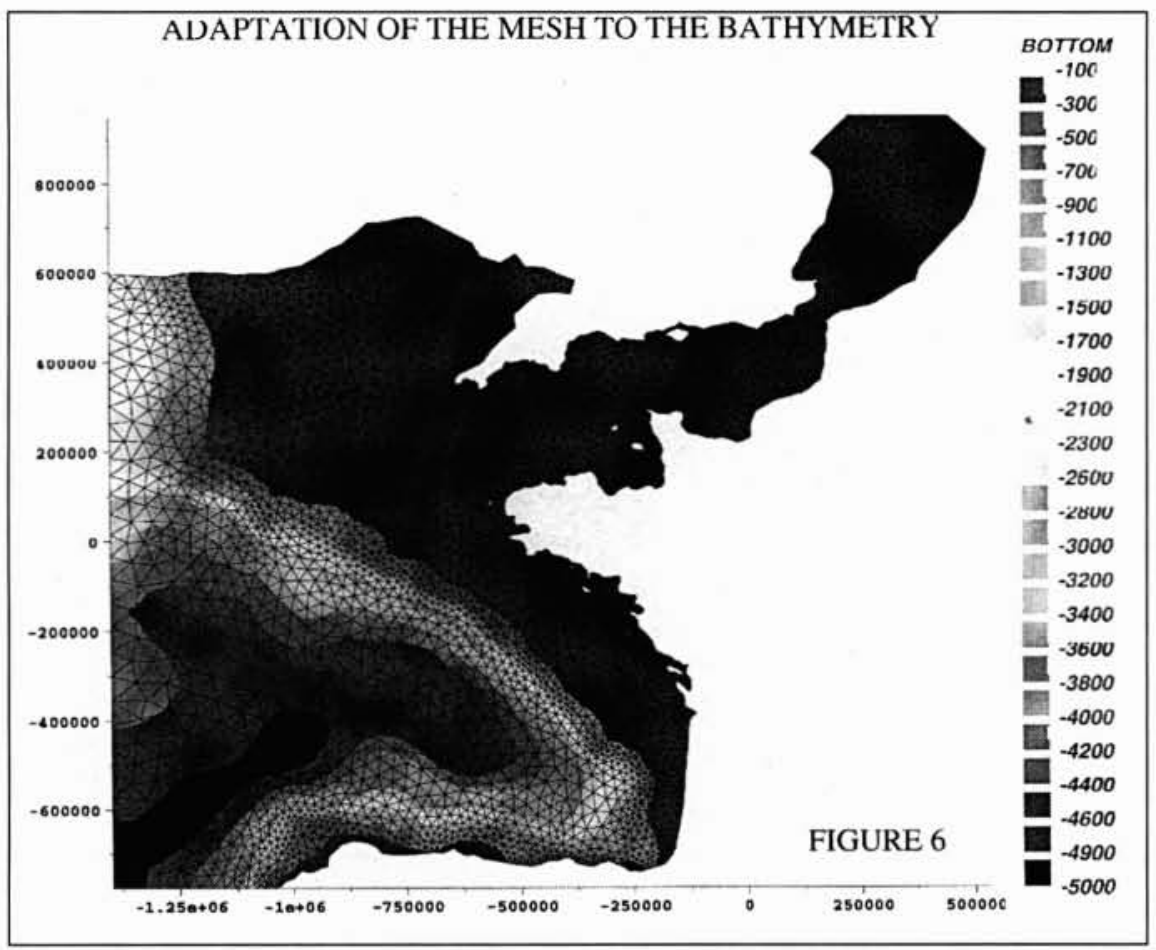

1. Adaptation of the mesh to the bathymetry. 
- propagation of long waves, the non-linear effects being taken into account;

- friction against the bottom:

- influence of the Coriolis force;

- sources and sinks of fluid and momentum within the domain;

- turbulence (various modelling options);

- sub- and super-critical flows, and transition from one of these regimes to the other one:

- influence of horizontal temperature or temperature variations on density;

- Cartesian or spherical coordinates for the large domains;

- consideration of the tide generating force for the large maritime domains;

- dry areas within the computation domain: tidal flats and flood plains;

- advection and diffusion of a pollutant, with source or sink terms:

- drogue monitoring and Lagrangian drifts;

- sills and submerged dikes.

The software has numerous fields of application. The following are noteworthy in the fluvial domain: the impact statements related to the building of such structures as bridges, sills, groins, the dam failures, the flood studies (see figure 2 ), the transport of both conservative and non-conservative tracers. In the maritime domain, the dimensioning of harbour works, the impacts of newly constructed submersible dikes or dredging, the impact of a coastal outfall discharge, the study of thermal plumes are particularly to be mentioned. Figure 3 displays the free surface in the Channel, with low tide at Mont Saint-Michel and in the Severn Estuary, the two hot spots of tides in Western Europe. TELEMAC-2D was also used in a number of special applications, just within its scope, such as the failure of industrial tanks, a snow avalanche falling into a reservoir, etc.

The scope of TELEMAC-2D can be assessed through its validation document. The latter was drafted as per the recommendations in the "Validation Task Force " of the major European hydraulics laboratories.

TELEMAC-2D solves, through a finite element method over non-structured grids consisting of triangles, the Barré de Saint-Venant equations in two horizontal space dimensions. These equations express the conservation of water mass (continuity equation) and the conservation of momentum in both horizontal space directions (dynamic equations) in every point of the computation domain. Furthermore, TELEMAC-2D can compute simultaneously, and in a coupled way, the advection and diffusion of any tracer (transport equation). Thus, the average vertical density of water can depend upon the local value of that tracer, as it happens, for instance, with salinity in the case of a study in an estuarial environment.

\subsection{TELEMAC-3D}

Some flows are vertically quite heterogeneous. Such is the case, for example, with a wind-driven stream within a closed domain, or with a bottom outfall discharging matters which are less dense than the surrounding environment, i.e. go up to the surface. A model solving these vertically averaged equations cannot handle these flows, and a three-dimensional model should therefore be implemented. The TELEMAC-3D digital model simulates the three-dimensional flows both in the seas and the rivers. It reproduces the surface movements (tide, river flood) and takes into account the processes acting upon the flow: water carried away by the drift of wind, the bottom friction, the Coriolis force, the turbulent mixing, the variations of water density resulting, for example, from salinity, temperature or suspended matter. TELEMAC-3D makes it possible to conduct a wide range of studies, among which the flows around a structure, the creation or destruction of a stratification, the dilution of an effluent or of the exhaust heat either from a nuclear plant or from any other industrial facility.

Two original applications, sponsored by the EEC, are related to the sediment transport. They deal with the modelling of currents induced by breaking waves and the simulation of

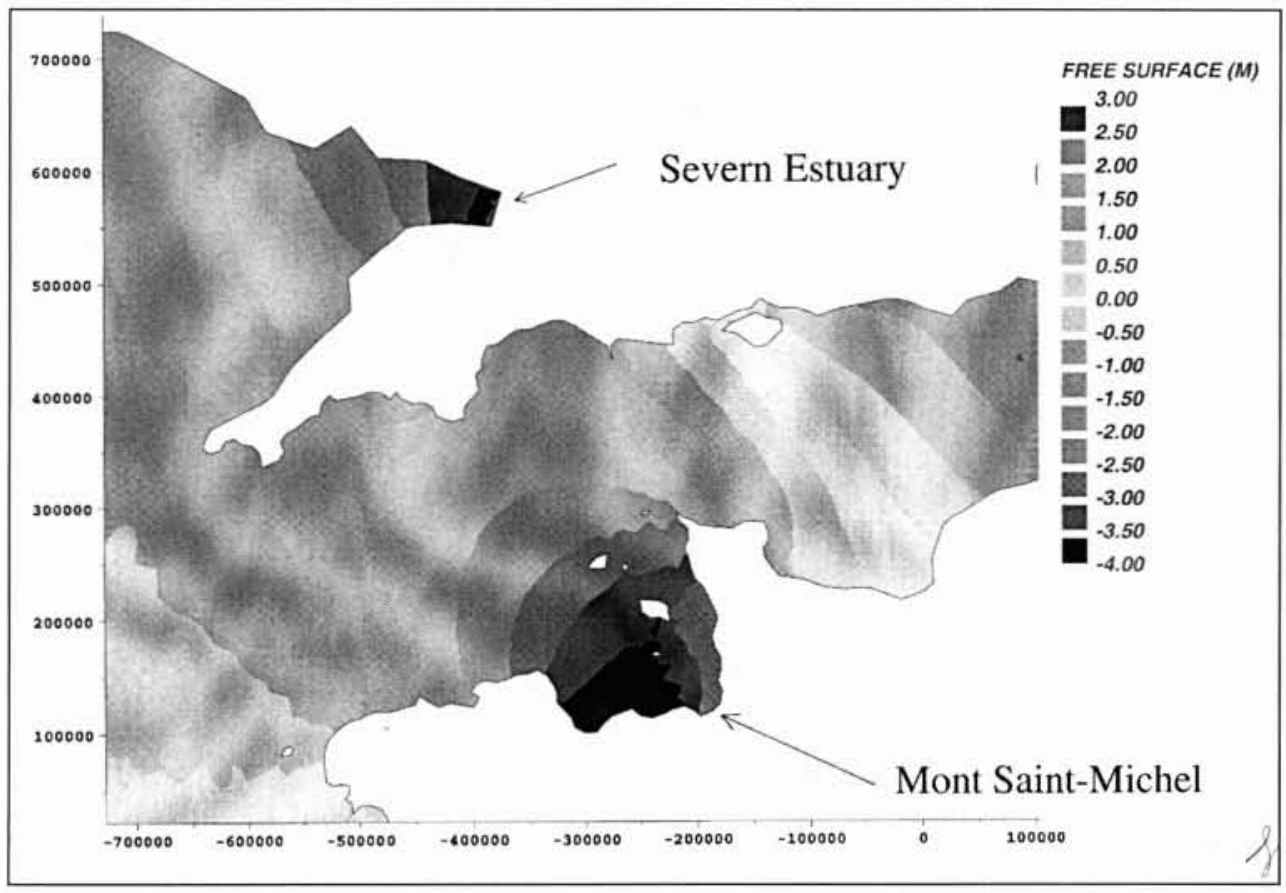

2. M2 Tidal wave in the channel. 


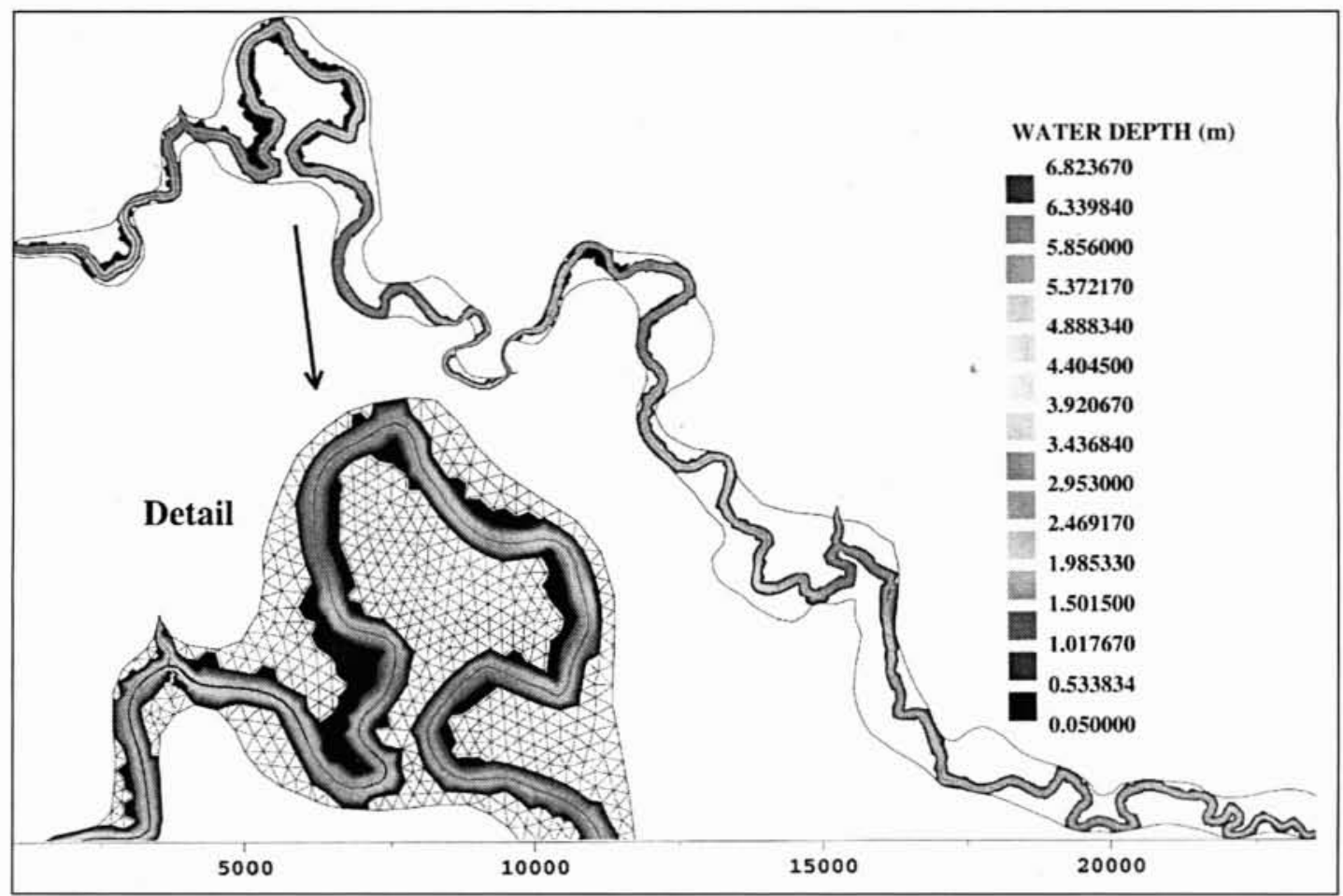

3. Computation of a flood in the river Severn.

(Courtesy

University of

Bristol). the transport of cohesive sediments within an estuary, including such specific behaviours as flocculation, bottom consolidation, mud transport.

TELEMAC-3D solves the Navier-Stokes equations with the hydrostatic pressure hypothesis. The transport-diffusion equations of one or several tracers with a source term can be coupled with the hydrodynamic equations through a state equation relating the density and the value of the tracer(s).

The turbulent mixing process is reproduced using a kepsilon-type model, a mixing length or a constant turbulent viscosity, depending on the applications.

The choices of numerical schemes were made in order to meet with two requirements, namely providing a high quality result at a reasonable cost. The algorithms are solved using a finite element method on a grid consisting of prisms. The numerical scheme for discretization in time uses an operator splitting method, so that each term of the equations can be processed through the optimal digital method, combining high performance, robustness and accuracy.

The programme was used many a time. The following examples are noteworthy:

- dilution of the termal plume from the Paluel, Penly, Gravelines and Flamanville nuclear power plants, on the Channel. That study completes the monitoring network already being locally operated;

- dilution of fresh water discharged from the Saint-Chamas hydroelectric power plant into the Berre lake, a body of brackish water since it is linked to the Mediterranean Sea;

- simulation of suspended sediment transport in the estuary of the river Loire in order to reproduce the tidal oscillation into the estuary, the salt intrusion and the mud transport;

- study of the route followed by the "Sea Wallnuts" (Ctenophores) by the Dunkirk harbour and the Gravelines nuclear plant.

\subsection{Wave modelling: ARTEMIS, TOMAWAC and COWADIS}

Wave modelling is one of the most complex issues in maritime hydraulics. Surface gravity waves are solutions of the Navier-Stokes equations, but solving these three-dimensional equations would only be possible over limited areas. Over larger computational domains, simpler equations are to be used and a loss of generality shall be accepted.

Apart from the Boussinesq model, which can also simulate waves, two system models, which process two different sets of simplified wave modelling equations, with different approximations and thus different application domains, are set out hereinbelow.

\subsection{ARTEMIS, Berkhof equations}

The Berkhof equation (or " mild slope equation ") is a two-dimensional horizontal equation which was developed by the Dutch scientist Berkhoff. The non-linear effects such as bottom friction and depth-induced breaking are taken into account. One assumes the waves have a low amplitude and the bottom varies slowly. ARTEMIS accurately models the diffraction, refraction and reflection in waters of any depth. It is well suited to studying harbour agitation, resonance in basins, etc. The Berkhof equation is written as follows:

$$
\nabla\left[\text { C. } C_{g} \nabla \phi\right]+\omega^{2} \frac{C_{g}}{C} \phi=0
$$

wherin $\Phi=\Phi(x, y)=\Phi_{r}(x, y)+\mathrm{i} \cdot \Phi_{i}(x, y)$ is the complex potential velocity, $\mathrm{C}$ and $\mathrm{Cg}$ denote the phase and group celerities respectively, $\omega$ denotes the wave angular frequency and $\nabla$ denotes the horizontal gradient operator. The boundary conditions are expressed in the following form :

$$
\frac{\partial \phi}{\partial \mathrm{n}}=\left(\mathrm{A} \cdot \phi_{\mathrm{i}}+\mathrm{B} \cdot \phi_{\mathrm{r}}\right)+\mathrm{i}\left(-\mathrm{A} \cdot \phi_{\mathrm{r}}+\mathrm{B} \cdot \phi_{\mathrm{i}}\right)-\mathrm{E}-\mathrm{i} \cdot \mathrm{F}
$$


The reals $A, B, E$ and $\Phi$ are determined by the types of selected boundary conditions. These conditions correspond either to open boundaries for which the waves come in or out, or to partly reflecting solid boundaries.

The Berkoff equation is obtained assuming that all the variables are periodical in time, at the angular frequency $\omega$. The computation of random uni- ou multidirectional waves, however, is made possible by discretizing the directional and frequential energy spectrum into a number of representative monochromatic waves with the same energy. The results are subsequently combined to obtain the energy level of the random waves.

\subsubsection{TOMAWAC and COWADIS, wave action equation}

TOMAWAC is a spectral third generation wave model. It models the main physical processes affecting surface gravity waves (wind-induced generation, propagation, refraction, dissipation induced by breaking and bottom friction...) for ocean domains or shelf seas. From wind fields which vary in time and are supplied as input, the programme computes the evolution of wave energy in both space and time, as well as the way such energy is distributed over wave frequencies and propagation directions. COWADIS is a simplified version of TOMAWAC where the first frequency moments of the energy spectrum only are computed.

\section{TOMAWAC:}

TOMAWAC can be used either for analytical purposes in order to simulate past storms (hindcasting) or for predictive purposes in order to compute the wave conditions by the coasts a few hours or days in advance (forecasting). TOMAWAC solves the equation of conservation of wave action spectral density in either Cartesian or spherical coordinates. That quantity is defined as the ratio of energy spectral density $E$ to the relative wave angular frequency $\sigma$. It is conserved (to within the energy generating or dissipating terms) in the general case wherein the waves propagate over a depth and a field of current which vary both in time and space.

TOMAWAC considers the following physical processes: generation by wind, dissipation by white-capping and bottom friction, non-linear interactions between frequency quadruplets. In addition, it includes the depth-induced breaking dissipation process. The interactions between frequency triplets are now also implemented, the latter interactions becoming of greater importance in shallow water.

The equation of energy density evolution is solved through a method of fractional steps in TOMAWAC:

- The advection step is handled through the method of characteristics. Since the advecting field is stationary, the characteristics are traced back only once at the beginning of the computation, the result is stored in memory. Thus, a quite fast advection scheme is obtained.

- The step of source term integration is solved through a semi-implicit scheme allowing comparatively large time steps.

\section{COWADIS:}

COWADIS was developped in order to deal with current engineering applications that does not need such a fine tool as TOMAWAC (wave propagation studies for example). The simplifications made in the equations solved (steady problems, loss of the frequency discretisation) allow strong improvements in terms of computation speed and memory requirement without spoiling the quality of the results.

\section{A few examples of applications}

- The heavy storms which occurred in the Channel and NE Atlantic Ocean in January, 1990 have been reproduced (see figure 4). The comparison of the model results with the field measurements is satisfactory.

- TOMAWAC was also used in the modelling of waves in the landing area of the NorFra pipeline, near Dunkerque. Wave propagation is particularly complex in that area which is featured by the presence of many sandbanks. Following the validation of the model upon a particular storm, a set of site-specific situations could be modelled using the programme.

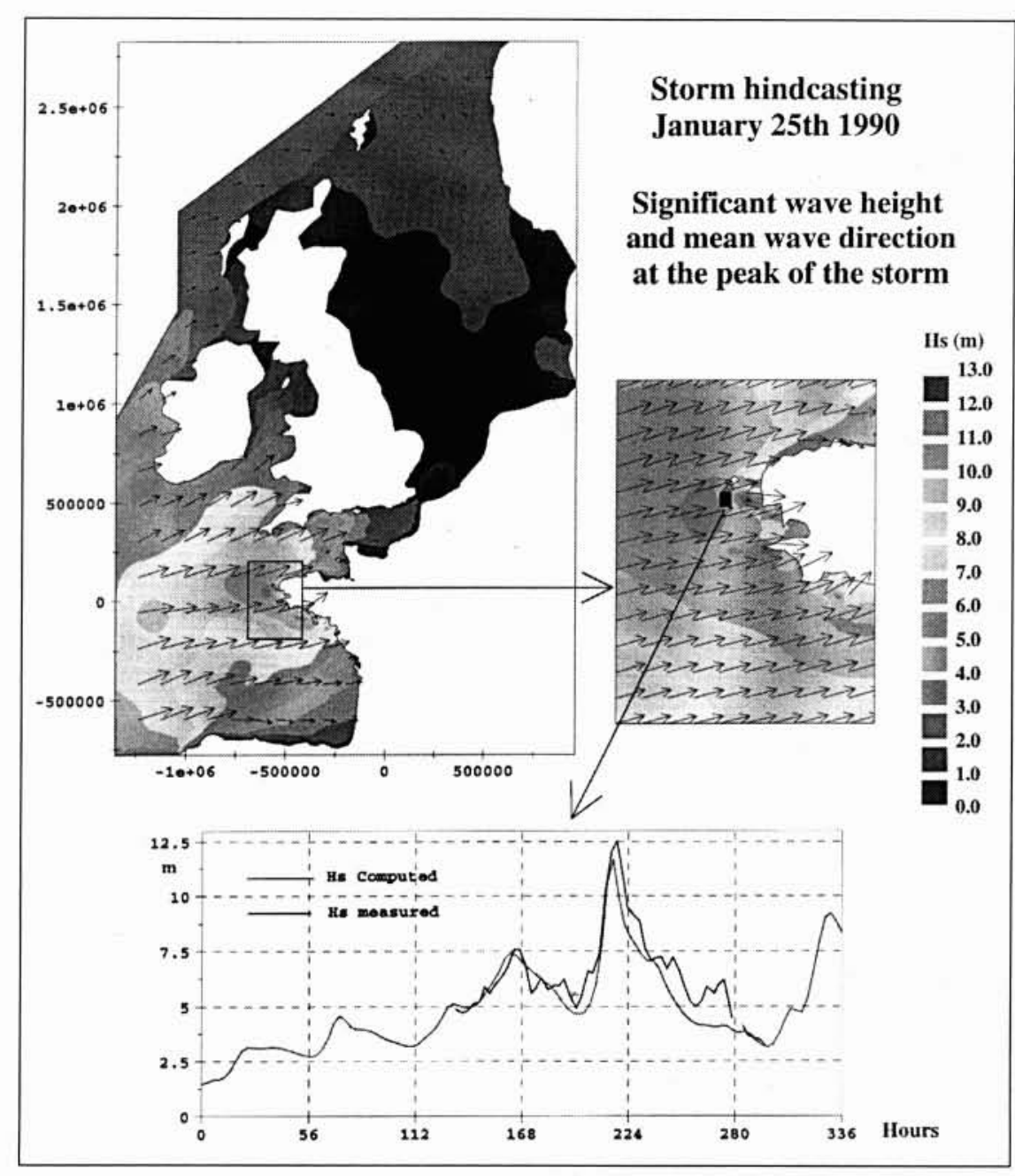

4. TOMAWAC - Third generation model. 


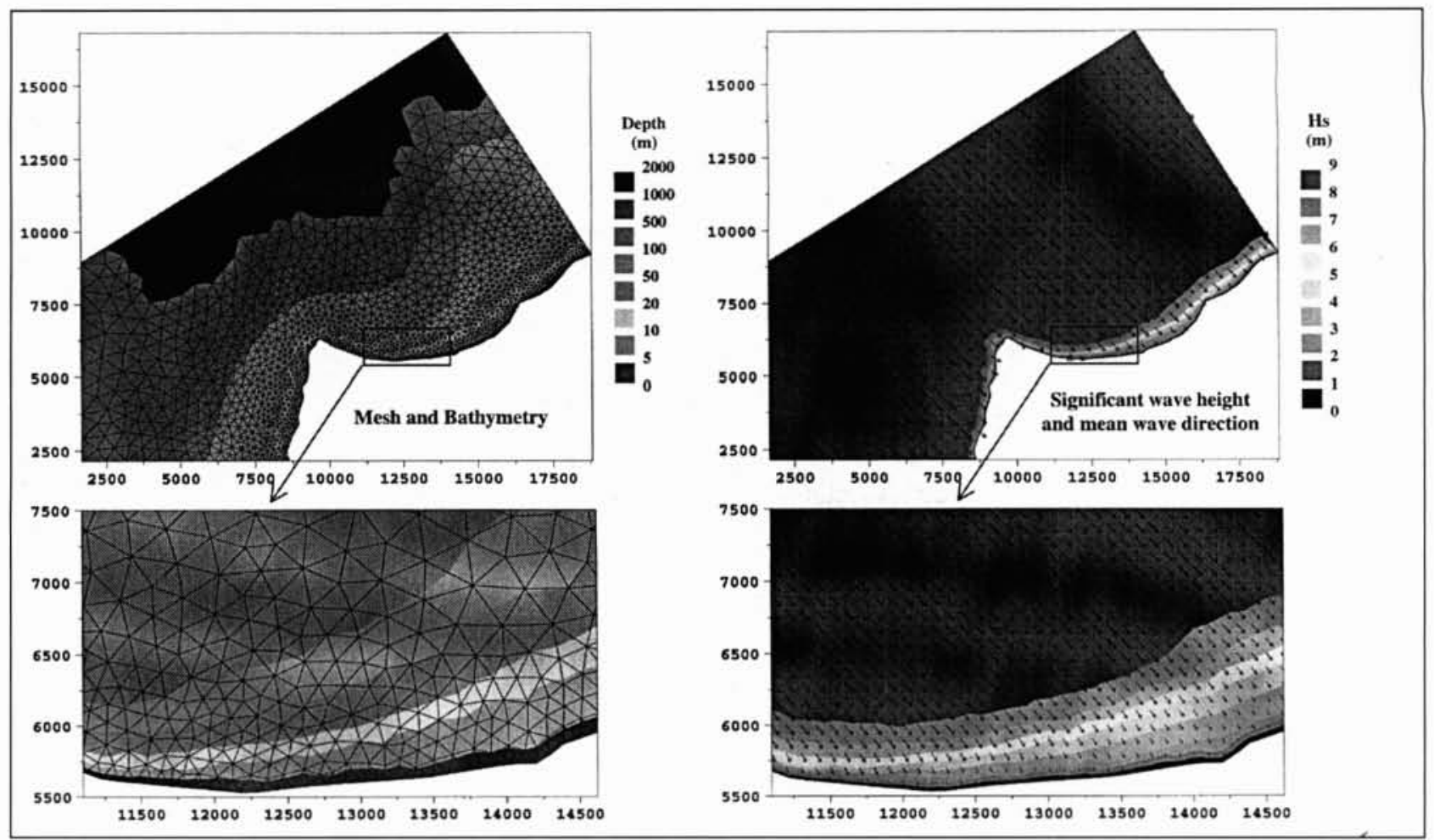

5. COWADIS - Computation of wave propagation in coastal areas.

- COWADIS was used in a wave propagation study for La Possession harbour in La Réunion Island (see figure 5).

\section{- 4.4 Bed load transport, SYSIPHE}

The sea and river currents, even moderate, induce a transport of sediments on the bottom.

In the absence of any human work, the resultant morphological changes are usually slow, through the mutual matching of flow and bottom topography as time elapses.

On the contrary, the construction of structures upsets that equilibrium and induces faster changes that are necessarily to be assessed, both to predict and minimize their impact and to ensure their good working order.

Nowadays, the high-quality modelling of currents makes it possible to determine the configurations of flows, even of complex ones, with an appropriate accuracy. Using experimentally determined solid transport formulas can then lead to the assessment of solid flow and bottom changes from the previously calculated hydrodynamic characteristics.

For that purpose, the LNH participated in the construction of the two-dimensional finite element code SYSIPHE, simulating the bed-load transport and the associated morphodynamic evolutions in both maritime and fluvial domains. The project was initiated by the french ministry of transportation (STCPMVN) and other partners are SOGREAH and the University of Compiègne. The SYSIPHE programme is now maintained and developped within the TELEMAC system.

SYSIPHE is not related to hydrodynamics as regards the equations and uses various transport formulas and the event (floods or tides) filtering techniques as developed in the LNH.
SYSIPHE can be coupled with TELEMAC-2D or 3D in order to compute the evolutions of bottom under the combined action of currents and waves. In the frame of the European programme MAST, the results achieved with this kind of coupling could successfully be compared with the experimental measurements of velocities and sand bottoms evolutions made in a channel of the Hannover University.

\subsection{Suspended sediment transport, SUBIEF-2D and TELEMAC-3D}

The SUBIEF-2D software is used for suspended sediment transport. This code also includes a modelling of water quality equations, to be explained in next section. Therefore, only the sediment transport aspect of SUBIEF will be described herein.

SUBIEF-2D includes a prediction model for suspended cohesive sediments, with erosion and deposition. Hydrodynamics and sedimentology are uncoupled: velocities and water heights are data and can be supplied by TELEMAC2D. SUBIEF solves in finite elements a vertically averaged equation of advection and dispersion, including the exchanges with the bottom. Deposition and erosion are related to the shear stress at the bottom. The consolidation of deposits is modelled as well.

The estuaries, as interfaces between fresh water and maritime environment, present a complex dynamics (tidal asymmetry, salinity intrusion, formation of a turbidity maximum) which requires a fine three-dimensional modelling. Such is the purpose of the developments realised in TELEMAC-3D within the framework of the MAST (Marine And Science Technology) project and in collaboration with the Hannover 
University. The modelled processes are the tidal currents, the buoyancy effects due to temperature, salinity or concentration and the suspended sediment transport. The equations of sediment transport and salinity are coupled with the equations of hydrodynamics. Turbulence is described by a mixing length model with damping functions. Flocculation, deposition and erosion, as well as the consolidation of deposited sediments, are taken into account.

Through these developments, TELEMAC-3D was successfully applied to the case of the Loire estuary, which combines the effects of a salt water wedge and mud transport.

\subsection{Water quality, SUBIEF-2D and SUBIEF-3D}

SUBIEF-2D is designed to assess the current-induced transport and dispersion of several elements which are usually termed "state variables". They are possibly suspended sediments, chemical or organic compounds, mere tracers on the stream. The state variables can either act upon one another or change according to source terms expressing chemical reactions, degradations, the action of external forces. The state variables and these possibly parametered source terms altogether form a water quality model.

A water quality model is defined in SUBIEF-2D through only one file. It includes the names of state variables, the various model parameters and the reactions written in a simple symbolic form which is close to the formalism as used by chemists. Upon the starting of SUBIEF-2D, this " water quality file" is interpreted by an interface which translates its content into Fortran language and integrates it into the source code of the software. Thus, the users have many opportunities to use, define and modify the water quality models without being necessarily familiar with programming in the TELEMAC system.

The available water quality models are related to bacteria, dissolved oxygen, adsorption/desorption of heavy metals or radioactive elements. All the features of SUBIEF-2D have recently been implemented in a 3-dimensionnal version called SUBIEF-3D.

\section{V $\square$ CURRENT DEVELOPMENTS}

Recent advances in the system are on the one hand parallelism for networks of workstations by means of domaindecomposition, on the other hand external code coupling between modules of the system, by means of a software called CALCIUM. Applications include parameter estimation in shallow-water equations (in this case 3 programs are run concurrently), and suspended sediment transport (in this case TELEMAC-2D and SUBIEF-2D are linked through CALCIUM, for alternate updating of the flow field and the topography). Parallelism and code coupling are based on the PVM (Parallel Virtual Machine) message passing language and linked programs need not be on the same machine. Developments on parallelism have been done within the framework of the PCECOWATER project. The PCECOWATER project (Parallel Computing of Environment COastal and lake shallow WATER dynamics) was part of the european ESPRIT project " Parallel Computing Initiative - II ". Partners in the project were ENEL, CRS4 and Politecnico de Milano in Italy, Reiter Ltd and Kemijoki Oy in Finland. The management of the PCI-II cluster of projects was ensured by the University of Catalunya. The goal was to provide the end-users with effective parallel simulation tools for large scale problems, on distributed memory systems. The endusers may be large companies with super-computers as well as consultants with limited computing capabilities, networks of workstations or PC's. With the technique of domain decomposition, which consists of sending a part of the computational domain to every processor, the speed-up obtained with parallelism is about 1.6 with 2 workstations; on real parallel machines, it can be a factor 6 with 8 processors (on IBM SP2), or 40 with 64 processors (case of CRAY T3E). Some large cases of TELEMAC-3D that could not be run on a workstation can now be computed using 2 machines, each one dealing with half of the computational domain.

An important application of TELEMAC for EDF is the computation of dam-break floodwaves. A recent study has

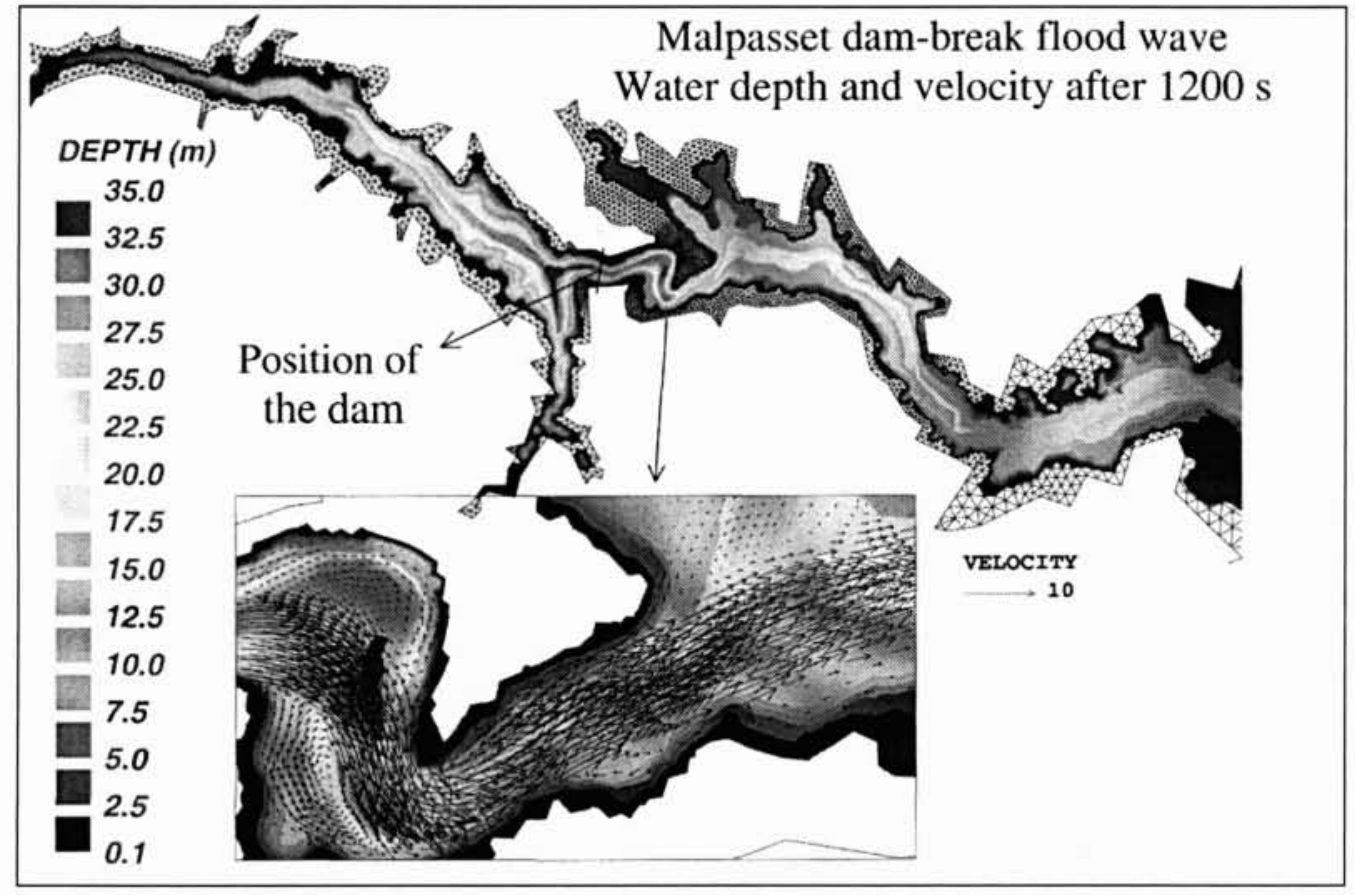

6. TELEMAC-2D computation of Shallow Water Equations - Malpasset dambreak flood wave.

Water depth and velocity after $1200 \mathrm{~s}$. 
been done with a 140000 element mesh, the machine used being a HP 9000 workstation. Such large cases will benefit from parallelism and the simulation of the Malpasset dambreak has been one of the test cases retained in the PCECOWATER project. Figure 6 shows the upper part of the Reyran valley and the flow downstream of the dam, $20 \mathrm{mn}$ after the dam-break.

Another very important development is also currently done in TELEMAC-3D: a new option without hydrostatic assumption is being prepared. Preliminary tests done at the University of Hannover are quite satisfactory and it will most probably be a big breakthrough. For the first time we shall have the full Navier-Stokes equations solved on a non structured grid and with a free surface. Depending on the computer time required, we can then think of using this program for computing waves, instead of resorting to simplified equations. A new deal in a near future is thus possible.

\section{VI $\square$ CONCLUSION}

The TELEMAC system is an example of what is now called hydroinformatics. Building such a system requires considerable efforts which can only be the result of an international collaboration. There exist but a few such systems in the world, and even less if one considers only non structured meshes. In the future, it is probable that we shall have, like in many other fields in industry, a concentration of efforts. International recognition and co-developments will thus play an important role. Whatever the efforts, one must have in mind that not all the expectations are yet met with. Sophisticated user interfaces, new numerical schemes, and the ever increasing power of hardware tend to give the idea that everything is or will soon be under control but we must admit that the demand for numerical simulation is far from being fully satisfied. Every new enhancement opens new fields of application, and raises new problems. In sedimentology or water quality, the research on physical laws is still an ongoing process and validation of numerics is thus even harder. Furthermore basic theoretical difficulties such as turbulence and hyperbolic equations remain and some may appear to be fundamental limitations. It is thus important that both developpers and end-users be aware of the state of the art, and this was the goal of this article.

\section{Remerciements}

Je remercie tous les participants au projet TELEMAC qui ont contribué à la rédaction de cet article : Catherine Le Normant, Denis Aelbrecht, Thierry Boulet. Thierry Denot. Mustafa Guesmia, Bruno Laborie, Frédéric Marcos, Soizic Péron, Damien Violeau, ainsi que Paul Bates à l'Université de Bristol et Patrick Sauvaget de la société SOGREAH. 